\title{
Encoding of forelimb forces by corticospinal tract activity in the rat
}

\section{Yi Guo, Richard A. Foulds, Sergei V. Adamovich and Mesut Sahin*}

Department of Biomedical Engineering, New Jersey Institute of Technology, Newark, NJ, USA

\section{Edited by:}

Yukio Nishimura, National Institute for Physiological Sciences, Japan

Reviewed by:

Chet T. Moritz, University of Washington, USA

Duk Shin, Tokyo Institute of

Technology, Japan

*Correspondence:

Mesut Sahin, New Jersey Institute of Technology, 323 MLK Blvd.,

Fenster Hall, Room 617, Newark,

NJ 07102, USA

e-mail: sahin@njit.edu
In search of a solution to the long standing problems encountered in traditional brain computer interfaces $(\mathrm{BCl})$, the lateral descending tracts of the spinal cord present an alternative site for taping into the volitional motor signals. Due to the convergence of the cortical outputs into a final common pathway in the descending tracts of the spinal cord, neural interfaces with the spinal cord can potentially acquire signals richer with volitional information in a smaller anatomical region. The main objective of this study was to evaluate the feasibility of extracting motor control signals from the corticospinal tract (CST) of the rat spinal cord. Flexible substrate, multi-electrode arrays (MEA) were implanted in the CST of rats trained for a lever pressing task. This novel use of flexible substrate MEAs allowed recording of CST activity in behaving animals for up to three weeks with the current implantation technique. Time-frequency and principal component analyses (PCA) were applied to the neural signals to reconstruct isometric forelimb forces. Computed regression coefficients were then used to predict isometric forces in additional trials. The correlation between measured and predicted forces in the vertical direction averaged across six animals was 0.67 and $R^{2}$ value was 0.44 . Force regression in the horizontal directions was less successful, possibly due to the small amplitude of forces. Neural signals above and near the high gamma band made the largest contributions to prediction of forces. The results of this study support the feasibility of a spinal cord computer interface (SCCI) for generation of command signals in paralyzed individuals.

Keywords: brain computer interfaces, corticospinal tract, time-frequency analysis

\section{INTRODUCTION}

Severe injuries at the cervical spinal cord can result in quadriplegia due to extensive paralysis of the body below shoulders. Braincomputer interfacing (BCI) is a technique to substitute for the lost command signals in these severely paralyzed individuals, using neural signals recorded from the brain. Brain-computer interfaces attempt to "read" the volitional information from various cerebral cortices, primarily those involved in planning and execution of the motor function. In the invasive versions, a large number of microelectrodes implanted in the brain parenchyma record single spike activity of local neurons to extract the volitional information. However, three decades of research has repeatedly concluded that stable recording of individual cell activities with microelectrodes have many technical challenges. The most significant problem is the layer of activated astrocytes that forms around the recording electrode (Biran et al., 2005) and makes it very difficult to follow single spikes over an extended period of time (Perge et al., 2013). Attempts have also been made to characterize the local field potentials and cortical surface recordings with non-penetrating electrodes as a source of volitional information (Schalk et al., 2008; Kubanek et al., 2009).

If we look at the big picture, the final pathway for all the motor control information processed in the brain is the descending tracts of the spinal cord before the signals reach the skeletal muscle. The corticospinal tract (CST) and the rubrospinal tract (RST) together make up the lateral descending system that controls the muscles of the extremities in all the mammals (Iwaniuk and Whishaw, 2000). These two tracts work synergistically (Whishaw and Gorny, 1996; Whishaw et al., 1998), although the relative importance of each may be different in different species. For instance, the magnocellular portion of the red nucleus, where the RST originates (Murray and Gurule, 1979), is larger, thus suggesting a greater role in rodents than in primates (ten Donkelaar, 1988). Unilateral lesioning of the medullary pyramid in rats impaired rotatory movements in the contralateral arm including limb aiming, pronation and supination but spared limb advancement, digit opening, arpeggio and grasping (Whishaw et al., 1998). The red nucleus lesions, in addition to producing similar impairments in rotatory movements, also attenuated arpeggio. Both lesions affected both proximal and distal musculature, however, even after combined lesions the rats were able to advance the limb, grasp food and withdraw the limb (Whishaw et al., 1998), suggesting that some components of skilled limb use are supported by descending neural pathways or spinal cord circuits other than the crossed RST or CST. Whishaw et al. (1998) concluded that rats with pyramidal tract lesions were more impaired in limb guidance than rats with red nucleus lesions, however the relative contributions of each tract were reversed in the control of the wrist and digits. In support of the importance of the CST in the rat, another study showed that the supination of the hand, while reaching for a vertical bar, was lost after a contralateral pyradoctomy (Carmel et al., 2010). Thus, the current research 
collectively suggests that both CST and RST are recruited in rats synergistically in the control of the forelimbs in a complementary way although the relative importance of each may be different depending on the behavioral context.

The number of fiber counts in the medullary pyramid varies between 73,000 and 150,000 in the rat [depending on whether light or electron microscopy is used (Leenen et al., 1985, 1989; Gorgels, 1990)]. Most of the fibers are slow conducting with diameters less than $1 \mu \mathrm{m}$ with the largest ones around $3.7 \mu \mathrm{m}$ (Dunkerley and Duncan, 1969). Being located in the most ventral side of the dorsal column of the rat spinal cord, the fine fibers of the CST can readily be contrasted with the large fibers of the more superficially placed ascending pathways of the dorsal column. The fast fibers reaching up to the speed of $19 \mathrm{~m} / \mathrm{s}$ (Mediratta and Nicoll, 1983), and presumably the largest ones, are very small in number in the rat CST (Casale et al., 1988).

The nature of the control information descending via the CST is not known; perhaps due to technical difficulties in recording neural signals directly from the tract in experimental animals. Instead, indirect observations are made though micro stimulation of the motor cortex in animals as well as transcranial magnetic stimulation in human subjects where the modulatory effect of CST is seen on the corresponding muscles. Micro stimulation of the primary motor cortex in rhesus macaques generated both facilitatory and suppression effects in both flexor and extensor muscles of the distal and proximal forearm (Park et al., 2004); which is a response presumably mediated through the CST. Transcranial magnetic stimulation of the supplementary and primary motor areas during isometric static hand force task produced EMG effects with similar amplitude and latencies recorded from the intrinsic hand muscles, suggesting that both cortical areas effectively control the spinal cord excitability (Entakli et al., 2013). Indeed, many areas of the neocortex send projections to the spinal cord through the CST. It is interesting to note that complete ablation of the sensorimotor cortex led to the loss of only about $50 \%$ of the axons in the medullary pyramid in rats, suggesting that remaining $50 \%$ of the myelinated axons come from areas other than the sensorimotor cortex.

Another method of gleaning information about the CST function during behavior is to correlate the single cell activity recorded from a brain region or scalp EEG to the muscle EMG signals. Frequency-domain analysis in humans (Conway et al., 1995) and monkeys (Baker et al., 1997) has shown that isometric muscle contractions at submaximal voluntary force levels are accompanied by synchrony in the beta band between the motor cortex and the EMG signals. This synchronization diminishes during dynamic muscle contractions and is replaced by a higher frequency band around $30-45 \mathrm{~Hz}$ (Omlor et al., 2007). Other groups have shown greater contributions in the high-gamma band, with electrocortigograms recorded from the primary motor or premotor cortices, to the upper extremity muscle activities in monkeys (Shin et al., 2012; Nishimura et al., 2013). The post-spike facilitation studies in awake monkeys demonstrated that the tonic discharges related to the static limb torque were more prominent in the corticomotoneurons (CM), cortical neurons that synapse directly on the spinal motor neurons, in contrast to the rubromotoneuronal cells (Lemon et al., 1986; Cheney et al., 1988).
[The evidence on existence of CM connections in the rat is controversial (Liang et al., 1991; Alstermark et al., 2004)].

As an alternative approach to BCIs, the lateral descending tracts of the spinal cord may be a potential site for taping into volitional motor signals. Due to the convergence of the cortical outputs into a final common pathway in the descending tracts of the spinal cord, neural interfaces with the spinal cord have a potential of being more compact than BCIs. In light of what we know about the CST, we assert that multi-electrode recordings made in this tract will be able to extract volitional motor information in behaving animals. As in the cortical approaches, the mechanical stability of the recording electrodes in the spinal cord is crucial for those source weights to be stable over time. In our previous work, recordings from the RST were found to be stable in signal amplitudes, but cross validation of regression coefficients for the forelimb kinematics between multiple trials was not successful (Prasad and Sahin, 2006a,b). The poor reproducibility of the regression coefficients was most likely caused by mechanical instability of the electrode interface, the wire and penetrating array electrodes. We then decided to test a flexible substrate, planar electrode array for this application. An electrode assembly with a stainless steel supporting frame was developed to restrict the movement of the array in the cord and extend the lifetime of the interconnecting ribbon cable that runs to the external connector. The well-defined positions of the contacts in the array also permitted sampling of the tract more uniformly than the wire electrodes. The level of success in predicting the forelimb isometric forces using the spinal signals was investigated. Partial results were published as a conference paper (Guo et al., 2013).

\section{MATERIALS AND METHODS ELECTRODE IMPLANT}

Polyimide substrate electrode arrays were custom-designed for this study (NeuroNexus, MI). The array consisted of $4 \times 8$ arrangement of 32 gold contacts with $15 \mu \mathrm{m}$ diameter and $80 \mu \mathrm{m}$ spacing. Every other column of contacts was offset with respect to the neighboring columns by $40 \mu \mathrm{m}$ to sample the CST cross sectional area more uniformly (see Figure 9). The array had a width of about $650 \mu \mathrm{m}$ and a thickness of $12 \mu \mathrm{m}$. A $2 \times 2 \mathrm{~mm}$ PDMS sheet $(127 \mu \mathrm{m}$ thick) was attached like a collar around the MEA ribbon cable exactly $1300 \mu \mathrm{m}$ from the tip (Figure 1). This attachment allowed precise control of penetration depth and enhanced the mechanical stability of the array by keeping it in vertical orientation in the cord.

A dorsal laminectomy was performed on C3-C4 segments under ketamine/xylazine anesthesia ( 80 and $12 \mathrm{mg} / \mathrm{kg}$ ). The modified electrode array was inserted into the dorsal column midsagittally with its contact side facing the preferred hand at $\mathrm{C} 4$. The point of entry was the posterior median sulcus of the cord and adjacent to the dorsal vein. The array was pushed into the median septum gently after making a small cut with a \#11 blade into the pia matter. A sweet spot exists between the two halves of the spinal cord where electrode penetration did not require much force. The CST occupies a region in the most ventral portion of the dorsal column extending from a depth of 1000-1300 $\mu \mathrm{m}$ measured from the pial surface in the cervical cord. The supporting PDMS attachment was slid under the dura after electrode insertion and 


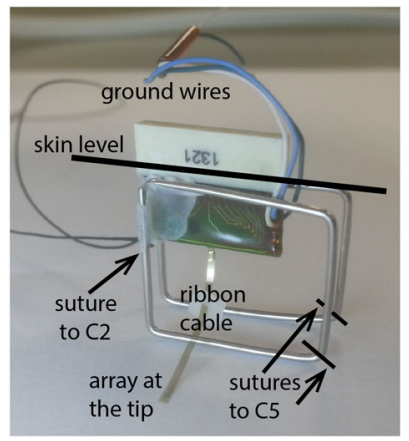

FIGURE 1 | A photo of the implanted electrode/connector assembly The electrode assembly was firmly fixed over the cervical spinal column by suturing the stainless steel wire frame to vertebral bones of C2 on the rostral and $\mathrm{C} 5$ on the distal side. The ground wires were placed over the spinal cord as a reference for neural recordings.

a small amount of cyanoacrylate was applied to the ribbon cable where it passes through the dura. A histological slide in Figure 2 depicts the track left behind by the array in the dorsal column after explantation.

The stainless steel wireframe $(\varnothing 0.75 \mathrm{~mm})$ shown in Figure 1 held the electrode assembly in place. The wireframe was tied to the spinous process through a hole made into the $\mathrm{C} 2$ vertebra on the rostral side and to the C5 on the caudal end with 6.0 silk sutures. The plastic connector was fixed to the frame with dental acrylic at a height that allowed some slack in the ribbon cable to reduce tension and hence the chronic trauma to the neural tissue. The reference electrode was placed on the dura next to the electrode ribbon cable and glued in place. The neck muscles and the skin were closed in layers around the connector using fine sutures. The plastic connector was protruding $\sim 5 \mathrm{~mm}$ through the skin opening. The gap around the connector was sealed with further dental acrylic, which also housed the nuts for anchoring the multi-channel wireless neural amplifier.

\section{ANIMAL TRAINING}

Six Long Evans rats (350-450 g) were used in this study. Food restricted rats were placed in a cage with a lever attached to a computer controlled haptic (with force feedback) device (Falcon, Novint Technologies, NM; see Figure 3). The lever was initially programmed to trigger release of $20 \mathrm{mg}$ sugar pellets on contact. Once the animal became familiar with the lever, the displacement required to trigger food reward was increased incrementally overtime to -14 or $18 \mathrm{~mm}$. The animals used both hands initially to press the lever in most cases. The lever position was switched to the corner of the box on the preferred hand side of the rat after this initial period of training. The animals then learned to do the task with their preferred hand. Behavioral training took 1-2 weeks prior to the implant surgery.

\section{DATA COLLECTION}

The haptic device (Falcon, Novint) produced 3D positional information at a rate of 1000 samples/s. A force/torque sensor (Nano

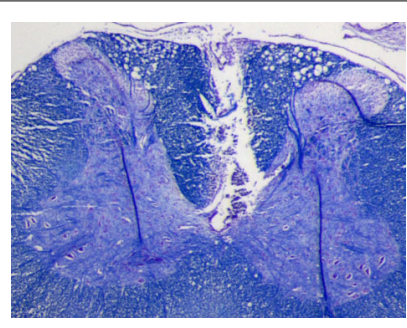

FIGURE 2 | Luxol fast blue stained transverse section of a cervical spinal cord from one of the rats. The lesion demarcates where the electrode array was implanted in the dorsal white matter. Because the tissue around was well attached to the substrate during three weeks of implantation time, it was ripped and stayed on the electrode during the explantation. Scale bar is $0.5 \mathrm{~mm}$.

17, ATI systems), integrated into the lever, measured the 3D forces applied to the contact pad at the tip of the lever by the rat's arm (Figure 3). Three dimensional force and position information were recorded from the lever. Of interest in the current study was the isometric portion following the lever press. The lever starts moving about $150 \mathrm{~ms}$ before the isometric portion begins. All data channels are backlogged continuously for $2000 \mathrm{~ms}$ until the lever press is detected. The isometric state, shown as the highlighted portion in the traces (see Figure 5), is achieved with a one-way spring-damper with high impedance to create a virtual boundary by the computer controlled haptic interface. The isometric interval ends when the rat lifts the hand from the lever to move toward the food reward at the end of the trial. The onset and offset time points of the isometric period are decided based on the vertical force being larger than a threshold $(\sim 0.11 \mathrm{~N})$.

Neural signals were amplified by a 31 channel wireless system (W32, Triangle Biosystems) before they were digitized at $16 \mathrm{kHz}$ simultaneously with the force data. Infrared cameras generated video logs at $10 \mathrm{fps}$ for quality assessment of the behavioral task. All data streams were synchronized using custom $\mathrm{C}++$ code.

Trained animals performed sessions of 50-200 lever presses (trials) per day until they lost interest in the sugar pellets. Trials with movement artifacts or aberrant postures were excluded from the analysis. Only one day of good data, i.e., one session, was found in each rat after removing all the trials with such defects, which precluded the investigation of multi-day applicability of regression coefficients.

\section{TIME-FREQUENCY ANALYSIS}

Neural signals were filtered in both directions in time (to cancel phase delay) using a 3rd order Butterworth band-pass filter at $75-425 \mathrm{~Hz}$ (Step A, Figure 4). Any component that exceeded $\pm 75 \mathrm{mV}$ (gain $=800$ ) in the filtered signals was considered an artifact, and upon detection $30 \mathrm{~ms}$ of the signal $(10 \mathrm{~ms}$ preceding it and $20 \mathrm{~ms}$ after) was substituted with zeros to remove it (Step B). Power spectral density was computed for each channel of filtered neural signals within a $40 \mathrm{~ms}$ moving window that shifted in $1 \mathrm{~ms}$ steps (Step C) using short time Fourier transform (STFT), which produced one Fourier coefficient per $25 \mathrm{~Hz}$ (total of 13 coefficients within $75-425 \mathrm{~Hz}$ band). Time signals representing the power variations in $25 \mathrm{~Hz}$ frequency bands were 


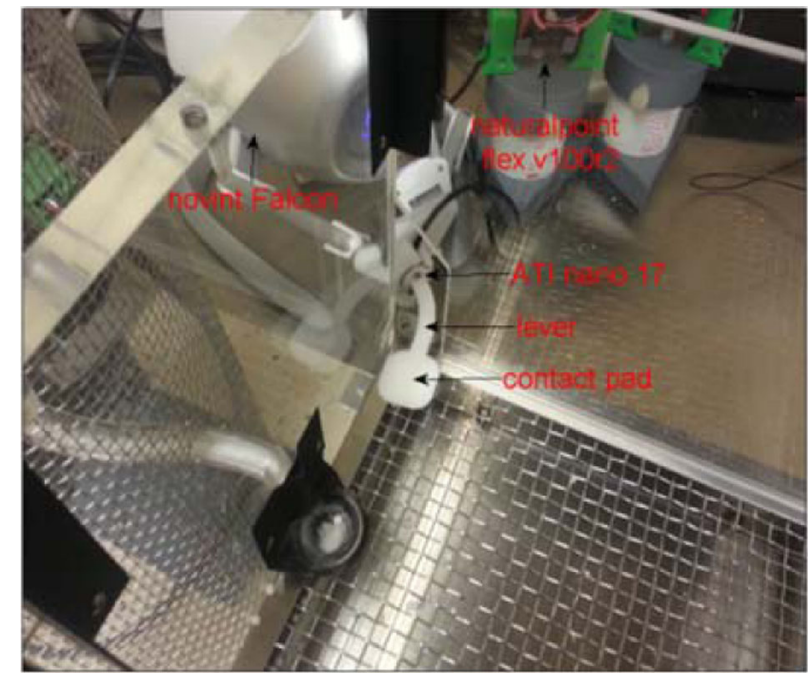

FIGURE 3 | A photo of the Falcon haptic manipulator with the force sensor and custom-design lever installed into a transparent training box. The picture shows the corner of the box from inside where a window was opened and the lever arm was brought in through the window. The rats were trained to push down on the contact pad with their preferred hand. The lever is switched to the opposite side of the box for a left-handed animal.

generated by taking the absolute value of FFT coefficients in this moving time window. A total of 403 channels of neural power signals were formed from 31 channels of neural signals (31 neural channels $\times 13$ frequency components for each channel) in each trial. A recursive averaging filter with a $400 \mathrm{~ms}$ time constant was applied first to account for the observed low-pass effect of the musculoskeletal system (Step D). A second filter with a sharper transition band (3rd order Butterworth low-pass filter, $\mathrm{fc}=8 \mathrm{~Hz}$ ) was applied to both the neural signals and the forearm forces in both directions in time to eliminate any phase lag (Step E). The force components above $8 \mathrm{~Hz}$ were considered as artifacts that could come from mechanical shaking of the lever.

\section{PRINCIPAL COMPONENT DECOMPOSITION}

The principal components (PCs) were computed and sorted in a descending order of variance. The PCs were grouped and regressed against each of the 3 -axes of the measured force as well as the magnitude (ABS) of the force vector (Step G in Figure 4). Regression weights of PCs in each group were pre-multiplied with pseudo inverse of corresponding columns of PCA score to generate weights for power signals, as follows:

Let $A$ be the transformation matrix from principle components $T$ back to power signals $X$ :

$$
\begin{gathered}
{[T] \times[A]=[X]} \\
{[T]=[X] /[A]}
\end{gathered}
$$

Forces can be estimated using PCs or power signals. Here $Z$ contains regression coefficients of principle components and $W$ is the

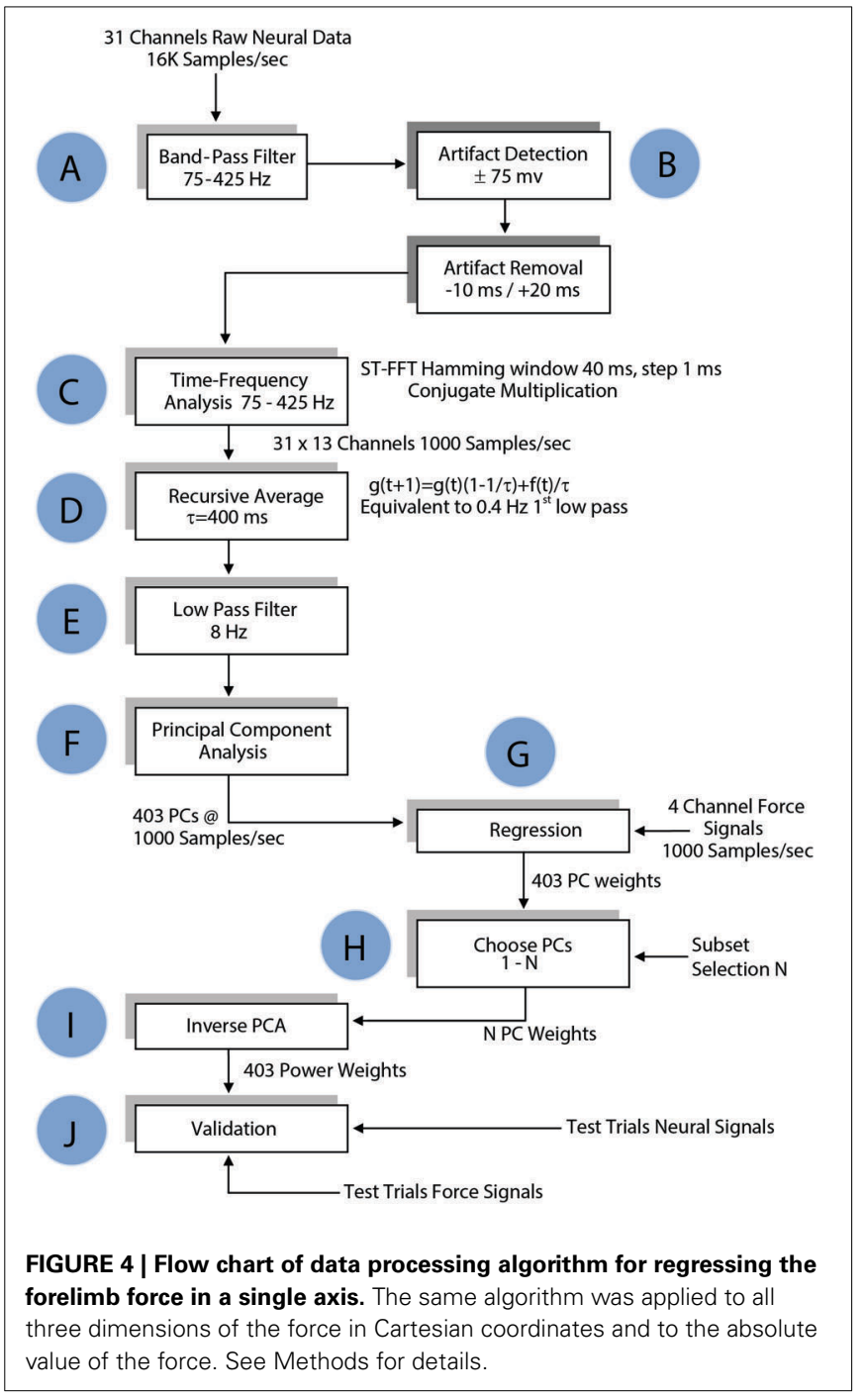

coefficients of power signals $X$ :

$$
[F]=[X] \times[W]=[T] \times[Z]=[X] /[A] \times[Z]
$$

Therefore weights of the power signal can be calculated from weights of principle components as follows.

$$
[W]=[I] /[A] \times[Z]
$$

$I$ is the identity matrix. Matrix division is used because $A$ may not be invertible.

\section{REGRESSION AND CROSS-VALIDATION}

Approximately one third of the trials in each session were held back for testing and the remaining trials were used for computation of the regression coefficients (training set). The test set data underwent the same time-frequency analysis as the training set. The weights for the power signals corresponding to each set of PCs calculated in the training set were applied to the test set (Steps I and J, Figure 4). The number of PCs in the group 
was increased incrementally to search for the best regression coefficients (Step H). Goodness of fit was measured by both the coefficient of correlation $(R)$ and the coefficient of determination, $R^{2}$ (Equation 5), for the entire session with the isometric force episodes taken from each trial and concatenated as a single episode. The weights that generated the best fit to the force in the training set were tested in the test set.

$$
R^{2}=1-\frac{\sum_{t}(F-\hat{F})^{2}}{\sum_{t}(F-\bar{F})^{2}}
$$

where the nominator is the sum of squares of prediction errors and the denominator is the variance of the measured force.

\section{RESULTS}

Three dimensional force and position information recorded from the lever is plotted in Figure 5 in a typical trial. The isometric state is shown as the highlighted portion in the traces. The isometric vertical force ( $Y$ in green) is the largest as expected for the lever pressing behavior. Nonetheless, small forces are recorded in the side-to-side ( $X$ in blue) and back-and-forth ( $Z$ in red) directions as well. The magnitude of the combined force vector (ABS) resembles that of the vertical force since it is the largest component. On the bottom plot, the rectified-filtered versions of the neural signals show different patterns in each channel, indicating spatially selective recordings of the neural sources via different electrode contacts. The neural channels contain signals that are moving at faster rates than the force traces, particularly with very few or no components that co-vary with the overall trajectory of the forces. This suggests that the spinal cord circuitry and the musculoskeletal system together may primarily be working as an integrator/low-pass filter on the descending control signals.

\section{OPTIMIZING THE REGRESSION COEFFICIENTS}

As mentioned above, the number of PCs was varied to search for the best force prediction in each session. Correlation and $R^{2}$ values increased (not monotonously but) steadily as additional PCs were included in the regression until a point of diminishing returns (vertical dash line in Figure 6). Adding more PCs over-fit the training set and yielded smaller correlation and $R^{2}$ values in the test set, which means that the set of PCs corresponding to the dashed line produced the best possible reconstruction. Furthermore, inclusion of additional trials into the set (e.g., compare the plots for 16 vs. 46 trials in Figure 6) improved the prediction when the optimal set of PCs is used. This further suggested that the reconstruction did not select features that over-fit the training data.

\section{RECONSTRUCTION OF FORELIMB FORCES}

Forelimb forces in all three directions were reconstructed by applying the best coefficients to the test set (Figure 7). Prediction was more effective for the absolute magnitude (not shown) and the vertical force than the other two directions in this and other animals, as indicated by the $R$ and $R^{2}$ measures (Table 1). In general, the reconstruction algorithm was more successful in predicting the average force amplitude than the rapidly changing
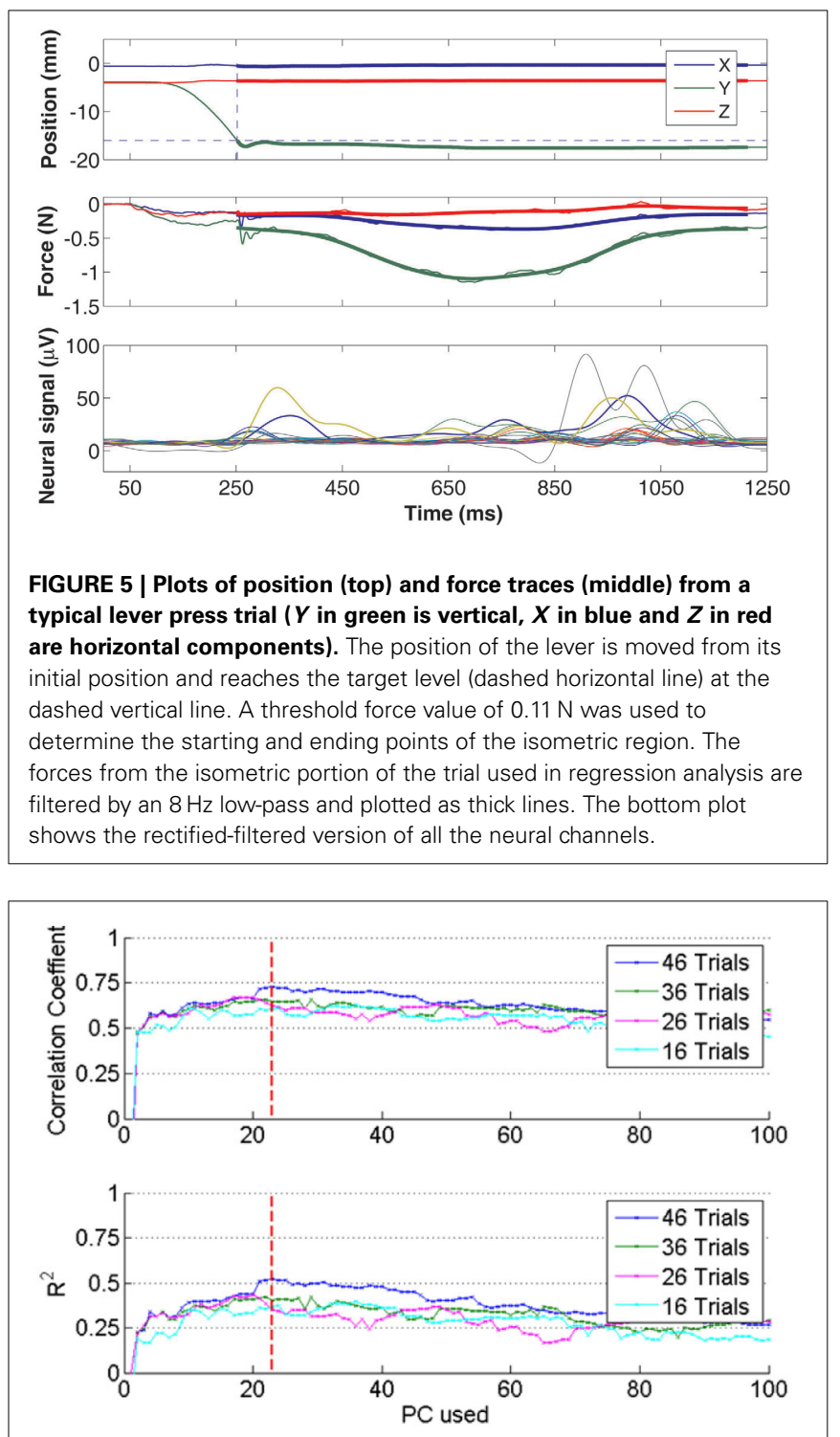

FIGURE 6 | The optimum regression coefficients were determined by increasing the number of PCs and the number of trials included into the analysis from a session. Top plot: correlation $(R)$, bottom: $R^{2}$ values obtained by applying the regression coefficients to the test set. Both $R$ and $R^{2}$ increased with increasing number of PCs up to the dashed vertical line. This procedure prevented over-fitting the data in the training set.

components of the forces in each trial. Coefficients of correlation between reconstructed and measured forces for individual trials (rightmost columns in Table 1) were not as high as the overall value for groups of trials in a training or test set. This is because the correlation coefficient for the entire test set accounts for the baseline changes from trial to trial but the correlation for an individual trial removes the baseline and only looks for the resemblance between phasic components of the predicted and actual force profiles within a trial. Thus, the $R$ values mostly represent the success in reconstructing the baseline level changes of the forces across multiple trials, although the phasic components are also reproduced in some of the trials. 


\section{FREQUENCY CONTRIBUTIONS}

Since the neural signals were separated into various frequency bands (Figure 4), it was possible to do back projection and determine the frequency components and the neural channels that were selected more often than others by the regression algorithm. The relative contributions of various neural channels and frequencies are plotted in Figure $\mathbf{8}$ for rat R1. The middle bar plot in Figure 8B corresponds to the $y$-force map in Figure 8A with all the contact contributions lumped together to show relative power contributions in different frequency bands with means and standard deviations calculated across trials. The bar plots for the other two directions ( $X$ and $Z$ ) were produced from similar maps. It is

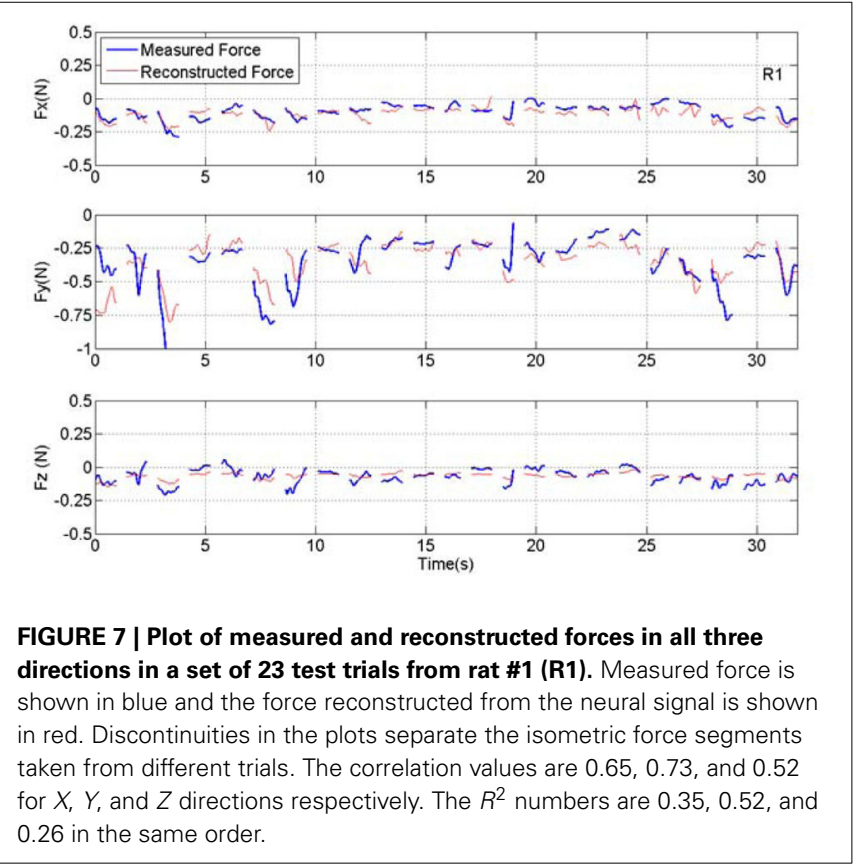

interesting to note that lower frequencies contributed more to the predicted force in all three dimensions. There is a local maximum around $300 \mathrm{~Hz}$ in the $X$ and $Y$ axes. Frequency contributions were calculated as mean power across all contacts averaged in time, i.e., over trials, and then converted into percentage by taking the power in each frequency band and dividing it by the total power. The small standard deviation bars, which were calculated on the percent power over multiple trials, suggest that the frequency contributions are relatively stable across multiple trials and somewhat similar in all three directions in this session. We limited the analysis retrospectively to frequencies below $425 \mathrm{~Hz}$ since percent contributions above this frequency were negligibly small. Components below $75 \mathrm{~Hz}$ had to be disregarded due to movement artifacts and $60 \mathrm{~Hz}$ contamination.

\section{SPATIAL DISTRIBUTION OF NEURAL SOURCES}

The spatial organization of the neural channels (i.e., contacts) on the array was also determined by back projection. Figure 9 illustrates the signal strengths (in all frequencies) by each contact on the array for all directions of force in rat R1. Interestingly, certain contacts were selected much more frequently than others and these contacts are located mostly near the caudal end of the array (left side) for all three directions of force. No single contact dominates as a signal source and not all the contacts make a significant contribution. It is encouraging to note for a neural interface that the standard deviations (shown as rings around contacts) are not very large, which suggest spatial stability of the neural sources that are selected by the algorithm across multiple trials in this session.

\section{GROUP RESULTS}

Force reconstruction plots (from the test sets) in the remaining rats of this study are shown in Figure 10. Only the vertical forces are plotted for brevity. In each implant, the vertical force amplitudes were predicted by the algorithm with a correlation coefficient that is above 0.58 . The duration of the isometric lever holding and the force profiles during each trial were substantially

Table 1 | Group statistics of test trials.

\begin{tabular}{|c|c|c|c|c|c|c|c|c|c|c|c|c|c|c|}
\hline \multirow[t]{2}{*}{ RAT } & \multirow[t]{2}{*}{ TRAIN COUNT } & \multirow[t]{2}{*}{ TEST COUNT } & \multicolumn{2}{|c|}{ TEST Lengths (ms) } & \multicolumn{4}{|c|}{ TEST $\mathbf{R}$} & \multicolumn{4}{|c|}{ TEST R2 } & \multicolumn{2}{|c|}{ TEST $\mathbf{R}$ by Trial } \\
\hline & & & MEAN & STD & $x$ & $Y$ & $z$ & ABS & $x$ & $Y$ & $z$ & ABS & MEAN & STD \\
\hline 1 & 46 & 23 & 905 & 111 & 0.65 & 0.73 & 0.52 & 0.72 & 0.35 & 0.52 & 0.26 & 0.52 & 0.24 & 0.56 \\
\hline 2 & 38 & 18 & 1015 & 575 & 0.60 & 0.64 & 0.53 & 0.64 & 0.36 & 0.39 & 0.24 & 0.38 & 0.20 & 0.65 \\
\hline 3 & 34 & 18 & 1923 & 758 & 0.56 & 0.62 & 0.51 & 0.60 & 0.30 & 0.36 & 0.25 & 0.35 & 0.39 & 0.42 \\
\hline 4 & 51 & 26 & 2433 & 89 & 0.59 & 0.67 & 0.43 & 0.66 & 0.35 & 0.44 & 0.17 & 0.42 & 0.63 & 0.25 \\
\hline Mean & 45.33 & 22.67 & & & 0.56 & 0.67 & 0.47 & 0.66 & 0.31 & 0.44 & 0.21 & 0.43 & & \\
\hline STD & 20.16 & 9.33 & & & 0.16 & 0.07 & 0.08 & 0.09 & 0.14 & 0.10 & 0.06 & 0.12 & & \\
\hline \multicolumn{2}{|c|}{ Weighted } & & & & 0.60 & 0.69 & 0.48 & 0.68 & 0.35 & 0.47 & 0.21 & 0.46 & & \\
\hline
\end{tabular}

Correlation (R) and $R^{2}$ statistics of regression in the test set in each rat of the study. Middle columns show the statistics when all the trials in the session are concatenated into one long trial for regression. The last two columns contain mean \pm STD of correlations calculated for each trial separately, while the regression was performed again on the concatenated version. 


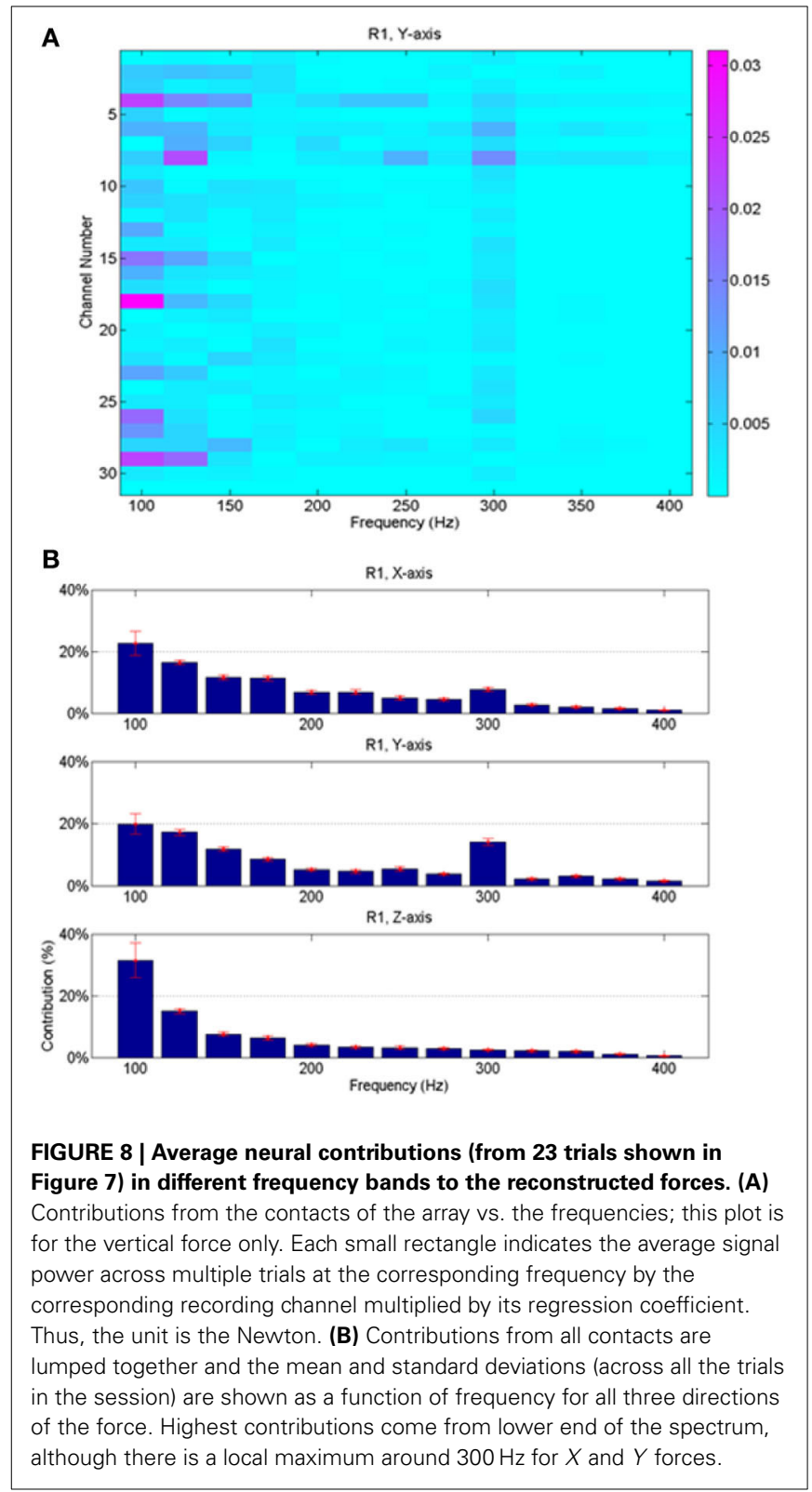

different (Test lengths column in Table 1). Moreover, each animal employed somewhat different strategies in its lever pressing behavior, as observed in video records. Therefore, the force data profiles did not appear to be stereotypical and thereby allowing a large area of the parameter space to be visited.

The frequency band contributions to the vertical force predictions are shown in Figure $\mathbf{1 1}$ for all the remaining animals. The mean and standard deviations are calculated across all the trials in the test sets. As in Figure 8, most of the signal power comes from the lower frequencies, except in R4 where there is a peak around $300 \mathrm{~Hz}$. Again, the standard deviations are small showing band limited signal contributions vary only slightly across multiple trials in each animal.

The spatial locations of the neural sources are depicted in Figure 12 for the remaining rats of this study. Only the vertical

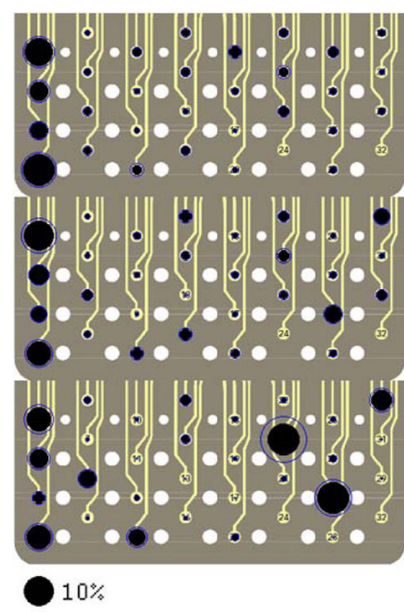

FIGURE 9 | Signal contributions from individual neural channels superimposed on three images of the electrode array in Rat 1. Each image contains percent contributions for one direction of the forelimb force ( $X, Y$, and $Z$, top to bottom). The sizes of the filled, black circles indicate average percent contributions across multiple trials in the session and the rings around the circles show the standard deviations. The large black circle on the bottom represents a contribution of $10 \%$. Contacts 24 and 32 were not used.

force maps are shown for brevity. Relatively small standard deviations, particularly for the contacts with large percent contributions, increase the confidence level in the reproducibility of the neural source locations within the spinal cord. The map does not reveal a preference in the dorsoventral direction that would point to a certain depth where most neural sources controlling the forelimb muscles might be located in the spinal cord cross-section. That is, the plots do not support the presence of somatotopic organization in the CST. The map looks very different in each animal, although some variation is expected since it is virtually impossible to implant the array in the same position in each animal and there are most probably some anatomical differences between the animals. In general, the contributions distribute across many contacts and even the largest contributions do not go above $10 \%$, with one exception in R2.

\section{STATISTICS OF RECONSTRUCTION}

Group statistics are summarized in Tables 1 and 2. The number of trials in the training and test sets are shown in the left most columns of Table 1 . The best correlation and $R^{2}$ values were obtained with R6, and the lowest coefficients with R5. Both measures were higher for the vertical force $(Y)$ and the magnitude of the force vector (ABS) than that for the other two horizontal directions ( $X$ and $Z$ ) in all subjects. The vertical correlation varied between 0.58 and 0.77 with a mean \pm STD of $0.66 \pm 0.07$ $(N=6)$. Similarly, the $R^{2}$ value for the vertical force changed between 0.33 and 0.59 with mean \pm STD of $0.43 \pm 0.10(N=6)$. The $R^{2}$ values are lower because this measure looks for an exact match between the actual and reconstructed forces, unlike the correlation coefficient which quantifies only the waveform similarity. Both measures for the ABS force were very close to that of the vertical force, presumably because the vertical force was the 


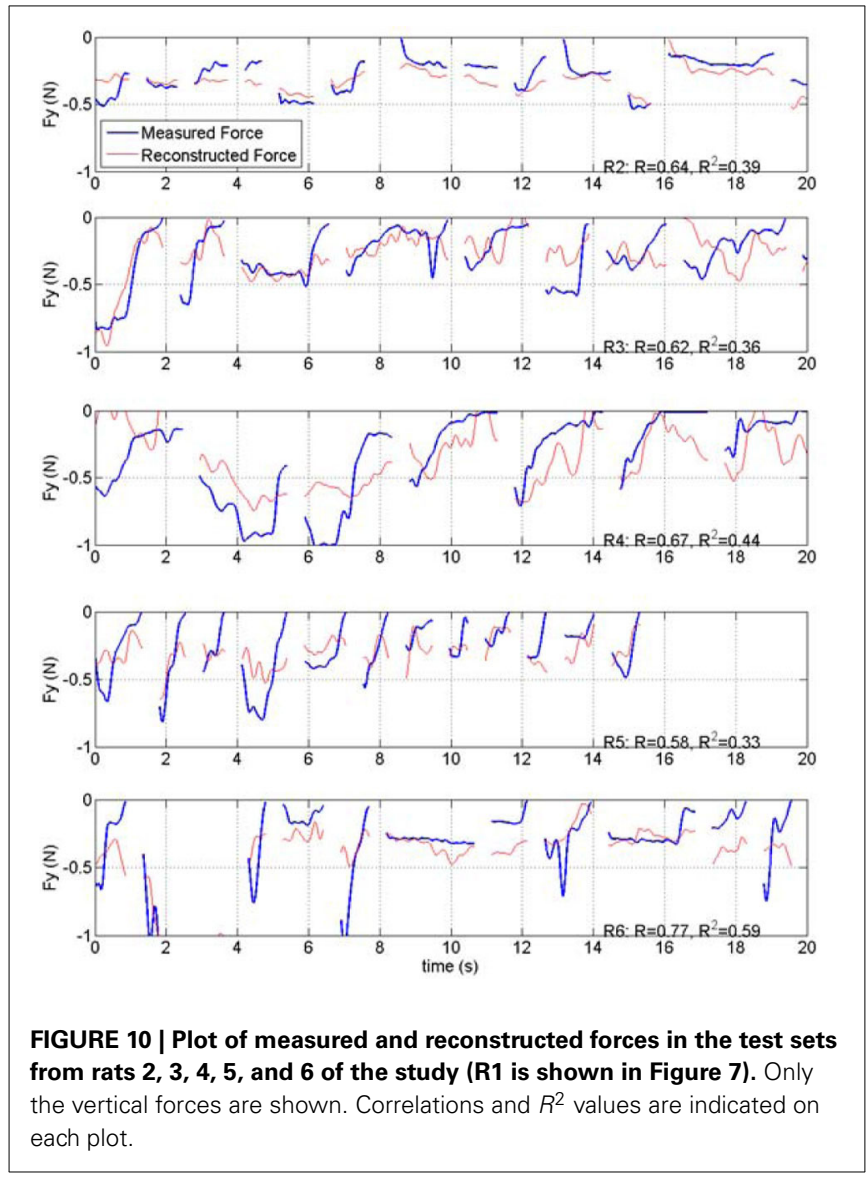

dominating component in the force vector. The last row in the table contains the mean values weighted by the number of trials in each test set. The weighted means are slightly higher because the largest test sets produced better predictions, especially in R5.

The mean correlations and $R^{2}$ values are presented in Table 2 from entire sessions including the training and test sets. These numbers illustrate that the regression algorithm performed only slightly better in the training set than it did in the test set, another evidence showing that the regression was not an over-fit to the data.

\section{DISCUSSION}

\section{FREOUENCY ANALYSIS}

Time-Frequency analysis (Cai et al., 2000) was invaluable in separating the neural signals that are most relevant to the forearm forces. Short Time Fourier Transform allowed multiple timevarying signals to be generated from a single physical channel. A single physical electrode can carry information from different neural sources in different frequency bands, and extraction of these sources into different channels was useful to improve the prediction. It is important to note that greater availability of signals required a larger training set. Additional trials were required to accurately estimate the extended set of coefficients.

The persistency of relative signal contributions to the force in various different frequency bands (Figures 8, 11) can be interpreted in different ways. This may be because the neural signals
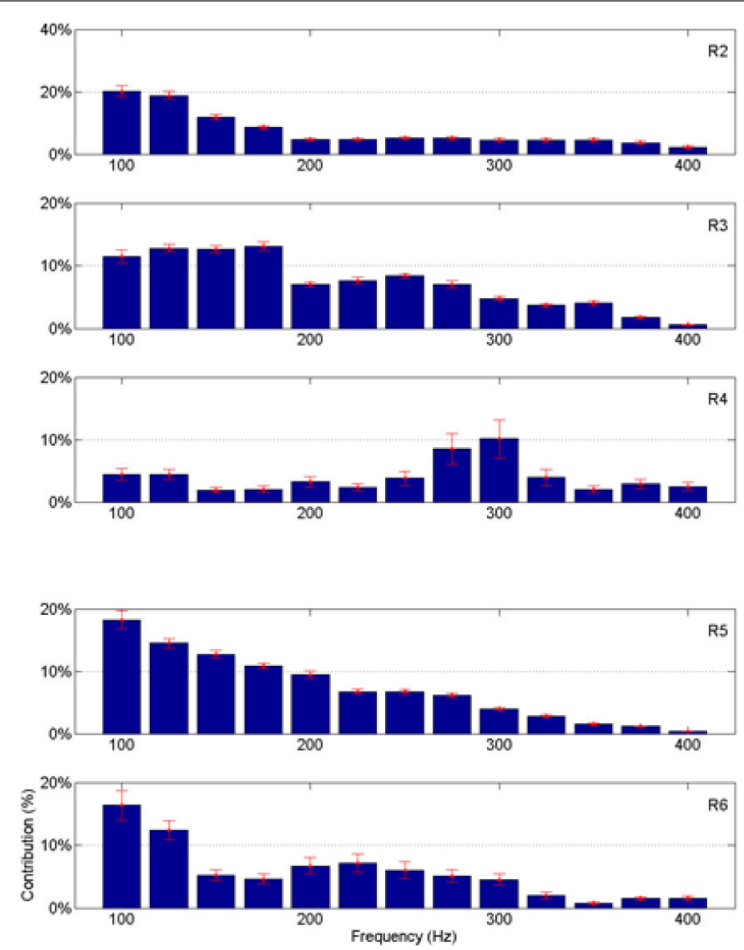

FIGURE 11 | Percent neural contributions to the vertical force $(Y)$ in different frequency bands for the rats $\mathrm{R} 2$ through $\mathrm{R} 6$ (top to bottom).

are composed of individual action potentials that have stereotypical shapes, which implies similar frequency components. However, the multi-unit neural signals usually occupy a frequency band starting around $300 \mathrm{~Hz}$ and reach up to a few $\mathrm{kHz}$. The entire spectrum may have moved to lower frequencies in these recordings because of the small size spectra of the fibers in the rat CST. The fact that the frequency spectrum of the raw neural activity was reaching up to $1 \mathrm{kHz}$ and even higher argues against this interpretation that the algorithm was selecting multiunit signals. Individual action potentials were observed to last less than a millisecond in the raw data. The fundamental frequencies and harmonics of single spikes should be above $1 \mathrm{kHz}$. The large contributions below $200 \mathrm{~Hz}$ may be interpreted as local field potentials. This raises the question of possible contamination from the gray matter neurons into the recorded activity. However, the largest neural activities are not recorded from the most ventral contacts in Figure 12, as one would expect if the array is too close to the gray matter in the dorsal column. Therefore, the most plausible explanation is that the low frequency components represent the firing rate of axons within the white matter. Mean firing rates of individual neurons in the primate motor cortex peak around 50 pps during behavior (Grammont and Riehle, 2003), a much smaller value than the frequency components contained in a single action potential. Even smaller frequencies can be present in the signals due to modulation of neural spike rates in time. Unfortunately, components below $75 \mathrm{~Hz}$ had to be discarded in this study to ensure that $60 \mathrm{~Hz}$ contaminations were completely suppressed. It is possible that the frequencies selected by our algorithm are the harmonics of those slower signal components. 


\section{FACTORS ON REGRESSION SUCCESS}

Compared to brain-computer interfaces where single spike activities are used to predict the forelimb or arm kinematics, the spinal method should be able to access signal with much richer volitional content. Nonetheless, it may still not be possible to collect all the forelimb related activity from the CST because of the limited recording range of the electrode array. The dorsoventral extent of the electrode is approximately $300 \mu \mathrm{m}$ and it matches the size of the CST in the rat. However, the majority of the fibers are smaller than $3.7 \mu \mathrm{m}$, as measured at the pyramids (Mediratta and Nicoll, 1983), (thus densely populated in a small cross sectional area of the dorsal column) and the number of recording

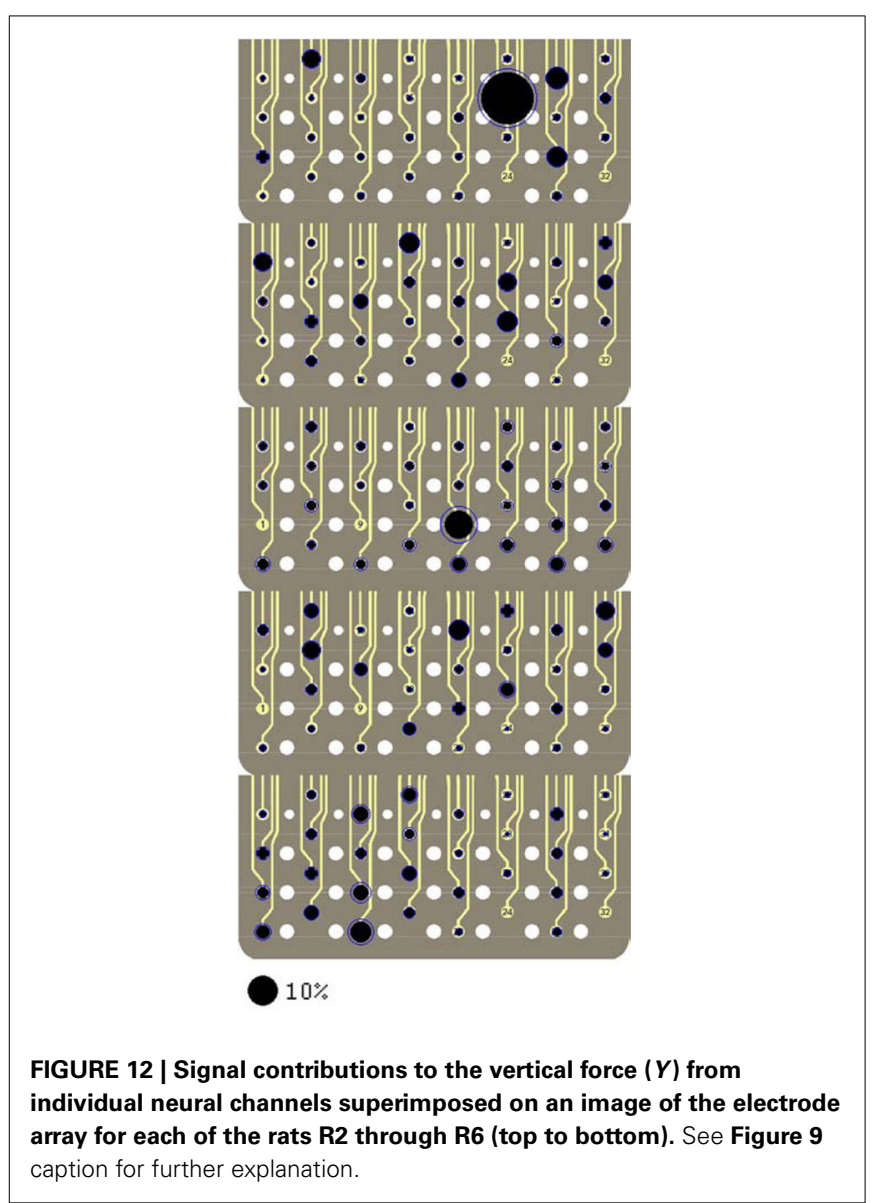

contacts in the array is too small compared to the number of neural sources even if a small percentage of axons become active simultaneously at any time instant. This issue of under sampling the available neural sources can be resolved to a large extent with much denser arrays and smaller contacts.

Force prediction could also be limited by the fact that the RST activity was not accessed in this study. The RST plays an important role in voluntary movements both in rodents and primates (Jarratt and Hyland, 1999; Iwaniuk and Whishaw, 2000; Webb and Muir, 2003). The RST is also known to take over the function of an injured CST (Belhaj-Saif and Cheney, 2000). A second array implanted in the RST can demonstrate the predictive power of this tract in comparison to the CST in different behavioral contexts in future experiments.

Accounting for the neural and muscle activation delays had negligible effects on the success of reconstructions. This may be explained by small propagation delays in neural conduction and the fact that very fast changing force components were taken out by low-pass filtering. The myelinated descending fibers reach up to velocities $\sim 19 \mathrm{~m} / \mathrm{s}$ and the mean velocity is about $11.4 \mathrm{~m} / \mathrm{s}$ (Mediratta and Nicoll, 1983), where the latter may be an overestimation in the cited study because of the tendency of electrical stimulations to recruit the larger and hence faster fibers first. Nevertheless, because the distance to the muscle is only in the order of centimeters, most of the delay can be attributed to muscle activation. The delay from the stimulation of cervical gray matter to the forelimb force initiation was less than $50 \mathrm{~ms}$ (unpublished results) in anesthetized rats in our laboratory. Because the forelimb forces did not contain very fast changes accounting for the neural conduction delay did not have a substantial effect on regression results.

\section{RECORDING ELECTRODES}

Two other types of electrodes were tested previously in our laboratory and the performance of flexible electrode arrays was deemed superior to both for this application. Utah type penetrating electrodes can generate severe neural damage in small animals due to their rigid substrate not conforming around the spinal cord (Rousche and Normann, 1998). Flexible electrode array also offered an additional benefit of recording from multiple sites in the same sagittal plane. The well-defined contact positions allowed a more uniform sampling of the CST activity in the mid-sagittal plane. Single wire electrodes had a tendency to disintegrate (Prasad and Sahin, 2006b) faster than the flexible

Table 2 | Group statistics of all trials.

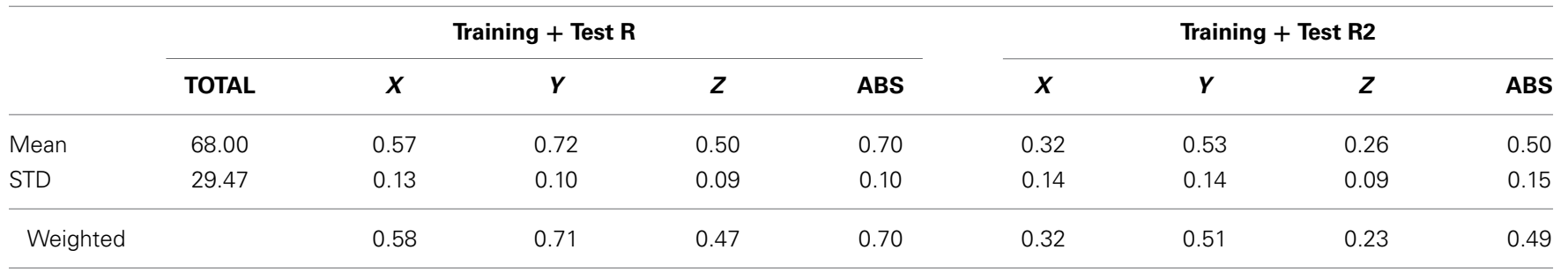

Correlation (R) and $R^{2}$ statistics in all the trials (both training and test sets) lumped together from all rats to show that the performance of the algorithm was comparable (and not superior) to that of test sets. 
electrode arrays even when wires were bundled within a silicone tube. Furthermore, the relative positions of wire electrodes were very difficult to control during implantation.

\section{CONCERNING A SPINAL CORD COMPUTER INTERFACE}

The long term objective of the study is to show the feasibility of extracting volitional components from the proximal side of the severed axons (at some distance from injury site) which were once serving the distal muscles. It should be noted that, for a spinal cord-computer interface, the targeted axons for recording in the proximal spinal cord are not only those that used to project to the upper limbs. Any neural component that is under volitional control can be utilized as a command signal in control of a prosthetic arm or other smart aids.

Needless to say, demyelination or degeneration of proximal axons would compromise the quality and information content of the targeted signals. Fortunately, the time course of retrograde degeneration in the lateral CST is much slower in humans than in rodents and a significant portion of the fibers is preserved even years after injury (Bronson et al., 1978; Fishman, 1987). Fishman (1987) reported from 12 patients post-mortem (carefully selected from a population of 343) who had an injury no lower than T10 and the shortest survival time of two years from the time of trauma. Spinal cord sections within a few spinal segments of the injury were grossly depleted of CST axons, however, patients with high thoracic to low cervical lesions had normal appearing CST by the high cervical levels regardless of post-injury times. Also, in rats transected at T8, a majority of the axons stopped dying back four weeks after injury at about $2.5 \mathrm{~mm}$ from the site and at 16 weeks the mean distance at which terminal bulbs were found was only $4 \mathrm{~mm}$ (Seif et al., 2007). These reports in animals and human cadavers strongly suggest that the descending signals should be available for long term recordings within the cervical CST, although no reports of such recordings are found in the literature to our knowledge.

\section{CONCLUSIONS}

This study demonstrated that volitional information contained in the CST about the forelimb forces can be accessed in the rat using flexible substrate array electrodes. The flexible MEAs may be a good choice to achieve a mechanically stable neural interface in the spinal cord. These results support the supposition that spinal cord-computer interfaces can eventually be built for subjects with spinal cord injury to operate manipulators with relatively little training.

\section{ACKNOWLEDGMENTS}

This study was supported by grants from NIH/NINDS (5R01NS072385) to Mesut Sahin, NIH/NICHD (R01HD58301) to Sergei V. Adamovich, National Institute on Disability and Rehabilitation Research Grant (H133E0511-06) to Richard A. Foulds, and a graduate fellowship to Yi Guo from New Jersey Commission on Spinal Cord Research. Special thanks to Sinan Gok for helping with data collection.

\section{REFERENCES}

Alstermark, B., Ogawa, J., and Isa, T. (2004). Lack of monosynaptic corticomotoneuronal EPSPs in rats: disynaptic EPSPs mediated via reticulospinal neurons and polysynaptic EPSPs via segmental interneurons. J. Neurophysiol. 91, 1832-1839. doi: 10.1152/jn.00820.2003

Baker, S. N., Olivier, E., and Lemon, R. N. (1997). Coherent oscillations in monkey motor cortex and hand muscle EMG show task-dependent modulation. J. Physiol. 501(Pt 1), 225-241. doi: 10.1111/j.1469-7793.1997.225bo.x

Belhaj-Saif, A., and Cheney, P. D. (2000). Plasticity in the distribution of the red nucleus output to forearm muscles after unilateral lesions of the pyramidal tract. J. Neurophysiol. 83, 3147-3153.

Biran, R., Martin, D. C., and Tresco, P. A. (2005). Neuronal cell loss accompanies the brain tissue response to chronically implanted silicon microelectrode arrays. Exp. Neurol. 195, 115-126. doi: 10.1016/j.expneurol.2005.04.020

Bronson, R., Gilles, F. H., Hall, J., and Hedley-Whyte, E. T. (1978). Long term post-traumatic retrograde corticospinal degeneration in man. Hum. Pathol. 9, 602-607. doi: 10.1016/S0046-8177(78)80143-9

Cai, L. Y., Wang, Z. Z., and Zhang, H. H. (2000). [A surface EMG signal identification method based on short-time Fourier transform]. Zhongguo Yi Liao Qi Xie Za Zhi 24, 133-136.

Carmel, J. B., Kim, S., Brus-Ramer, M., and Martin, J. H. (2010). Feed-forward control of preshaping in the rat is mediated by the corticospinal tract. Eur. J. Neurosci. 32, 1678-1685. doi: 10.1111/j.1460-9568.2010.07440.x

Casale, E. J., Light, A. R., and Rustioni, A. (1988). Direct projection of the corticospinal tract to the superficial laminae of the spinal cord in the rat. J. Comp. Neurol. 278, 275-286. doi: 10.1002/cne.902780210

Cheney, P. D., Mewes, K., and Fetz, E. E. (1988). Encoding of motor parameters by corticomotoneuronal (CM) and rubromotoneuronal (RM) cells producing postspike facilitation of forelimb muscles in the behaving monkey. Behav. Brain Res. 28, 181-191. doi: 10.1016/0166-4328(88)90095-2

Conway, B. A., Halliday, D. M., Farmer, S. F., Shahani, U., Maas, P., Weir, A. I., et al. (1995). Synchronization between motor cortex and spinal motoneuronal pool during the performance of a maintained motor task in man. J. Physiol. 489(Pt 3), 917-924.

Dunkerley, G. B., and Duncan, D. (1969). A light and electron microscopic study of the normal and the degenerating corticospinal tract in the rat. J. Comp. Neurol. 137, 155-183. doi: 10.1002/cne.901370204

Entakli, J., Bonnard, M., Chen, S., Berton, E., and De Graaf, J. B. (2013). TMS reveals a direct influence of spinal projections from human SMAp on precise force production. Eur. J. Neurosci. 39, 132-140. doi: 10.1111/ejn.12392

Fishman, P. S. (1987). Retrograde changes in the corticospinal tract of posttraumatic paraplegics. Arch. Neurol. 44, 1082-1084. doi: 10.1001/archneur.1987.00520220078021

Gorgels, T. G. (1990). A quantitative analysis of axon outgrowth, axon loss, and myelination in the rat pyramidal tract. Brain Res. Dev. Brain Res. 54, 51-61. doi: 10.1016/0165-3806(90)90064-6

Grammont, F., and Riehle, A. (2003). Spike synchronization and firing rate in a population of motor cortical neurons in relation to movement direction and reaction time. Biol. Cybern. 88, 360-373. doi: 10.1007/s00422-002-0385-3

Guo, Y., Mesut, S., Foulds, R. A., and Adamovich, S. V. (2013). Corticospinal signals recorded with MEAs can predict the volitional forearm forces in rats. Conf. Proc. IEEE Eng. Med. Biol. Soc. 2013, 1984-1987. doi: 10.1109/EMBC.2013. 6609918

Iwaniuk, A. N., and Whishaw, I. Q. (2000). On the origin of skilled forelimb movements. Trends Neurosci. 23, 372-376. doi: 10.1016/S0166-2236(00)01618-0

Jarratt, H., and Hyland, B. (1999). Neuronal activity in rat red nucleus during forelimb reach-to-grasp movements. Neuroscience 88, 629-642. doi: 10.1016/S03064522(98)00227-9

Kubanek, J., Miller, K. J., Ojemann, J. G., Wolpaw, J. R., and Schalk, G. (2009). Decoding flexion of individual fingers using electrocorticographic signals in humans. J. Neural Eng. 6:066001. doi: 10.1088/1741-2560/6/6/066001

Leenen, L. P., Meek, J., Posthuma, P. R., and Nieuwenhuys, R. (1985). A detailed morphometrical analysis of the pyramidal tract of the rat. Brain Res. 359, 65-80. doi: 10.1016/0006-8993(85)91413-1

Leenen, L. P., Meek, J., Posthuma, P. R., and Nieuwenuys, R. (1989). Differences in the fiber composition of the pyramidal tract in two- and 14-month-old rats. Neuroscience 28, 635-643. doi: 10.1016/0306-4522(89)90010-9

Lemon, R. N., Mantel, G. W., and Muir, R. B. (1986). Corticospinal facilitation of hand muscles during voluntary movement in the conscious monkey. J Physiol. 381, 497-527.

Liang, F. Y., Moret, V., Wiesendanger, M., and Rouiller, E. M. (1991). Corticomotoneuronal connections in the rat: evidence from double-labeling 
of motoneurons and corticospinal axon arborizations. J. Comp. Neurol. 311, 356-366. doi: 10.1002/cne.903110306

Mediratta, N. K., and Nicoll, J. A. (1983). Conduction velocities of corticospinal axons in the rat studied by recording cortical antidromic responses. J. Physiol. 336, 545-561.

Murray, H. M., and Gurule, M. E. (1979). Origin of the rubrospinal tract of the rat. Neurosci. Lett. 14, 19-23. doi: 10.1016/0304-3940(79)95337-0

Nishimura, Y., Perlmutter, S. I., and Fetz, E. E. (2013). Restoration of upper limb movement via artificial corticospinal and musculospinal connections in a monkey with spinal cord injury. Front. Neural Circuits 7:57. doi: 10.3389/fncir.2013.00057

Omlor, W., Patino, L., Hepp-Reymond, M. C., and Kristeva, R. (2007). Gammarange corticomuscular coherence during dynamic force output. Neuroimage 34, 1191-1198. doi: 10.1016/j.neuroimage.2006.10.018

Park, M. C., Belhaj-Saif, A., and Cheney, P. D. (2004). Properties of primary motor cortex output to forelimb muscles in rhesus macaques. J. Neurophysiol. 92, 2968-2984. doi: 10.1152/jn.00649.2003

Perge, J. A., Homer, M. L., Malik, W. Q., Cash, S., Eskandar, E., Friehs, G., et al. (2013). Intra-day signal instabilities affect decoding performance in an intracortical neural interface system. J. Neural Eng. 10:036004. doi: 10.1088/17412560/10/3/036004

Prasad, A., and Sahin, M. (2006a). Multi-channel recordings of the motor activity from the spinal cord of behaving rats. Conf. Proc. IEEE Eng. Med. Biol. Soc. 1, 2288-2291. doi: 10.1109/IEMBS.2006.260854

Prasad, A., and Sahin, M. (2006b). Extraction of motor activity from the cervical spinal cord of behaving rats. J. Neural Eng. 3, 287-292. doi: 10.1088/17412560/3/4/005

Rousche, P. J., and Normann, R. A. (1998). Chronic recording capability of the Utah Intracortical Electrode Array in cat sensory cortex. J. Neurosci. Methods 82, 1-15. doi: 10.1016/S0165-0270(98)00031-4

Schalk, G., Miller, K. J., Anderson, N. R., Wilson, J. A., Smyth, M. D., Ojemann, J. G., et al. (2008). Two-dimensional movement control using electrocorticographic signals in humans. J. Neural Eng. 5, 75-84. doi: 10.1088/ 1741-2560/5/1/008

Seif, G. I., Nomura, H., and Tator, C. H. (2007). Retrograde axonal degeneration "dieback" in the corticospinal tract after transection injury of the rat spinal cord: a confocal microscopy study. J. Neurotrauma 24, 1513-1528. doi: 10.1089/neu. 2007.0323

Shin, D., Watanabe, H., Kambara, H., Nambu, A., Isa, T., Nishimura, Y., et al. (2012). Prediction of muscle activities from electrocorticograms in primary motor cortex of primates. PLoS ONE 7:e47992. doi: 10.1371/journal.pone.0047992

ten Donkelaar, H. J. (1988). Evolution of the red nucleus and rubrospinal tract. Behav. Brain Res. 28, 9-20. doi: 10.1016/0166-4328(88)90072-1

Webb, A. A., and Muir, G. D. (2003). Unilateral dorsal column and rubrospinal tract injuries affect overground locomotion in the unrestrained rat. Eur. J. Neurosci. 18, 412-422. doi: 10.1046/j.1460-9568.2003.02768.x

Whishaw, I. Q., and Gorny, B. (1996). Does the red nucleus provide the tonic support against which fractionated movements occur? A study on forepaw movements used in skilled reaching by the rat. Behav. Brain Res. 74, 79-90. doi: 10.1016/0166-4328(95)00161-1

Whishaw, I. Q., Gorny, B., and Sarna, J. (1998). Paw and limb use in skilled and spontaneous reaching after pyramidal tract, red nucleus and combined lesions in the rat: behavioral and anatomical dissociations. Behav. Brain Res. 93, 167-183. doi: 10.1016/S0166-4328(97)00152-6

Conflict of Interest Statement: The authors declare that the research was conducted in the absence of any commercial or financial relationships that could be construed as a potential conflict of interest.

Received: 14 December 2013; accepted: 17 March 2014; published online: 01 May 2014. Citation: Guo Y, Foulds RA, Adamovich SV and Sahin M (2014) Encoding of forelimb forces by corticospinal tract activity in the rat. Front. Neurosci. 8:62. doi: 10.3389/ fnins.2014.00062

This article was submitted to Neuroprosthetics, a section of the journal Frontiers in Neuroscience.

Copyright (c) 2014 Guo, Foulds, Adamovich and Sahin. This is an open-access article distributed under the terms of the Creative Commons Attribution License (CC BY). The use, distribution or reproduction in other forums is permitted, provided the original author(s) or licensor are credited and that the original publication in this journal is cited, in accordance with accepted academic practice. No use, distribution or reproduction is permitted which does not comply with these terms. 